Received: 2015.03 .27

Accepted: 2015.05.11

Published: 2015.08.22

\title{
Prevalence of and Risk Factors for Type 2 Diabetes Mellitus in Hyperlipidemia in China
}

Authors' Contribution

Study Design A

Data Collection B

Statistical Analysis C

Data Interpretation D

Manuscript Preparation E

Literature Search F

Funds Collection $C$
AB 1,2 Guang-Yu Chen

BC 3 Lui Li

CD 4 Fei Dai

$C D \quad$ Xing-Jian Li

BC 2 Xiao-Xin Xu

ABCDEFG 1 Jian-Gao Fan
1 Center for Fatty Liver Disease, Department of Gastroenterology, Xinhua Hospital, Shanghai Jiao Tong University School of Medicine, Shanghai, P.R. China

2 Research Center for Clinical Epidemiology, Shanghai Jiao-Tong University School of Medicine, Shanghai, P.R. China

3 Department of Chronic Disease, Shanghai Center for Disease Control and Prevention, Shanghai, P.R. China

4 School of Public Health, Shanghai Jiao-Tong University School of Medicine, Shanghai, P.R. China
Corresponding Author:

Source of support:

Background:

Material/Methods:

Results:

Conclusions:

MeSH Keywords:

Full-text PDF:
Jian-Gao Fan, e-mail: fattyliver2004@126.com

This project was funded by the State Key Development Program for Basic Research of China (2012CB517501), Shanghai Municipal Health Bureau Foundation (01zD001), Shanghai Science and Technology Commission Foundation (10411956300), and the 100 Talents Program of the Shanghai Board of Health (XBR2011007)

\section{with and without dyslipidemia.}

We conducted a cross-sectional survey including 14385 adults (aged 16 to 88 years) in Shanghai using a stratified, multistage cluster sampling approach.

Type 2 diabetes and hyperlipidemia were found in 1456 (10.1\%) and 4583 (31.9\%) subjects, respectively. Type 2 diabetes was more common in males $(11.4 \%)$ than in females $(9.2 \%, P<0.01)$, in the elderly $(>$ or $=65$ years, $22.5 \%)$ than in younger $(<55$ years, $<10 \%, P<0.01)$ individuals, and in urban $(12.8 \%)$ than in rural populations $(5.2 \%, P<0.01)$. Diabetes incidence was higher among patients with hyperlipidemia than in controls $(16.9 \% \mathrm{vs}$. $7.0 \%, P<0.01 ; \mathrm{OR}=2.72,95 \% \mathrm{Cl} 2.44-3.03)$. Compared with controls, the risk for diabetes in subjects with isolated hypertriglyceridemia, isolated hypercholesterolemia, and mixed hyperlipidemia increased 1.75 -fold (95\% $\mathrm{Cl} 1.53-1.99), 1.53$-fold ( $95 \% \mathrm{Cl} 1.17-2.01$ ), and 2.93-fold ( $95 \% \mathrm{Cl} 2.37-3.63)$, respectively. The fasting plasma glucose (FPG) and $2 \mathrm{~h}$-postprandial plasma glucose (2h-PG) increased with age in both sexes. The age- and sexadjusted FPG and $2 \mathrm{~h}-\mathrm{PG}$ levels in hyperlipidemia were significantly higher than in controls $(P<0.01)$.

A high prevalence of type 2 diabetes in hyperlipidemia patients exists in Shanghai. Hyperlipidemia is associated with elevated blood glucose levels and therefore requires prompt intervention for prevention and treatment of diabetes in patients with dyslipidemia.

Diabetes Mellitus, Type 2 • Hyperlipidemias • Risk Factors

http://www.medscimonit.com/abstract/index/idArt/894246

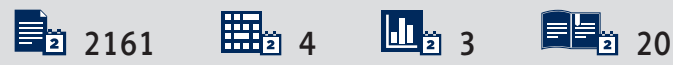




\section{Background}

Type 2 diabetes is one of the most common chronic conditions and its prevalence has increased continuously over the past decades. China's rapid economic growth, increased life expectancy, and altered lifestyle have significantly increased the prevalence of type 2 diabetes [1-3]. Dyslipidemia is a common comorbidity in diabetes [3]. In Asian and European epidemiological studies, hyperlipidemia is commonly associated with diabetes $[3,4]$. However, few, if any, epidemiological surveys have investigated the role of serum lipids in incident of type 2 diabetes in Shanghai, the biggest city of China [5].

Therefore, we carried out a subanalysis of dada of the Shanghai Dyslipidemia Study [6], using a cross-sectional survey to determine the prevalence of type 2 diabetes in patients with and without dyslipidemia. We assessed the risk odds of type 2 diabetes associated with different type of hyperlipidemia in the adult population of Shanghai.

\section{Material and Methods}

\section{Study design and participants}

The study is a stratified, multistage cluster sampling research involving 2 rural districts and 4 urban districts of Shanghai region. The study design, selection criteria, methodology, and participants' baseline characteristics have been detailed elsewhere [6]. Inclusion in the study was restricted to adults aged at least 16 years and registered as local residents. Participants with known type 1 diabetes, serious diseases, or pregnancy were excluded. Anthropometric indexes, including body weight, height, and waist and hip circumference, were measured. Body mass index (BMI, weight/height ${ }^{2}\left[\mathrm{~kg} / \mathrm{m}^{2}\right]$ ) and waist-to-hip ratio (WHR) were calculated from these measurements. The levels of serum triglyceride (TG), total cholesterol (TC), and high-density lipoprotein cholesterol (HDL-C) were measured. Participants were administered a standard 2-h 75-g oral glucose tolerance test (OGTT).

The study protocol, which is in accordance with the Declaration of Helsinki and the Ethical Guidelines for Clinical/Epidemiological Studies of the National Health and Family Planning Commission of the People's Republic of China, received ethics approval from the institutional review boards of all the participating institutions. All enrolled patients provided written informed consent.

Individual data were forwarded to the Epidemiology Unit of Shanghai Jiao Tong University for data analysis.

\section{Working definitions}

Type 2 diabetes as defined by the American Diabetes Association, is characterized by fasting plasma glucose (FPG) level $\geq 7.0$ $\mathrm{mmol} / \mathrm{L}(126 \mathrm{mg} / \mathrm{dl})$ or $2 \mathrm{~h}$-postprandial plasma glucose (2h-PG) level of at least $11.1 \mathrm{mmol} / \mathrm{L}(200 \mathrm{mg} / \mathrm{dl})$ during OGTT. Other parameters were defined as follows. Isolated impaired fasting glucose (IFG): FPG level, $5.6 \mathrm{mmol} / \mathrm{L}[100 \mathrm{mg} / \mathrm{dl}]-6.9 \mathrm{mmol} / \mathrm{L}$ [125 mg/dl], and 2h-PG level,<7.8 mmol/L [140 mg/dl]); Isolated impaired glucose tolerance (IGT): 2h-PG level, $7.8 \mathrm{mmol} / \mathrm{L}$ [140 mg/dl] $-11.0 \mathrm{mmol} / \mathrm{L}[199 \mathrm{mg} / \mathrm{dl}]$ and FPG level,<5.6 $\mathrm{mmol} / \mathrm{L}[100 \mathrm{mg} / \mathrm{dl}])$; and Combined IFG and IGT: FPG level, $5.6 \mathrm{mmol} / \mathrm{L}[100 \mathrm{mg} / \mathrm{dl}]-6.9 \mathrm{mmol} / \mathrm{L}[125 \mathrm{mg} / \mathrm{dl}]$, and $2 \mathrm{~h}-\mathrm{PG}$ level, $7.8 \mathrm{mmol} / \mathrm{L}$ [140 mg/dl]-11.0 mmol/L [199 mg/dl]) [7]. Previously diagnosed diabetes was identified by a valid questionnaire. Impaired glucose regulation was defined as either impaired fasting glucose or impaired glucose tolerance.

According to the criteria set by the National Cholesterol Education Program-Adult Treatment Panel III (NCEP-ATP III) [8], hyperlipidemia was classified into three phenotypes: isolated hypertriglyceridemia ( $\mathrm{TG} \geq 1.7 \mathrm{mmol} / \mathrm{L}$ and $\mathrm{TC}<6.2 \mathrm{mmol} / \mathrm{L}$ ); isolated hypercholesterolemia ( $\mathrm{TC} \geq 6.2 \mathrm{mmol} / \mathrm{l}$ and $\mathrm{TG}$ $<1.7 \mathrm{mmol} / \mathrm{L}$ ); and mixed hyperlipidemia ( $\mathrm{TG} \geq 1.7 \mathrm{mmol} / \mathrm{L}$ and TC $\geq 6.2 \mathrm{mmol} / \mathrm{L})$.

\section{Statistical analysis}

All analyses were performed using SPSS for Windows (version 13.0; SPSS Inc., Chicago, IL, USA). The significance of univariate differences was assessed by chi-squared and Student's ttests. Logistic regression analysis was performed to estimate the odds ratio (OR) of type 2 diabetes and $95 \%$ confidence intervals (Cls). We used logistic regression models to estimate the adjusted odds ratios (ORs) and 95\% confidence intervals $(\mathrm{Cl})$ for incident type 2 diabetes comparing three phenotypes of hyperlipidemia categories to the normal group. The data were adjusted for the multiple covariates. In the multivariate models, we included variables that might confound the relationship between hyperlipidemia and type 2 diabetes, which include: age (per 10-yr increment), sex, urban residence, waist circumference, BMI and phenotypes of hyperlipidemia. A P-value $<0.05$ was considered statistically significant and all values were two-sided.

\section{Results}

\section{Sample status}

A total of 17526 individuals were invited to participate in the study, of which 14401 participants completed the study, with an overall response of $82.2 \%$. After the exclusion of 12 subjects 


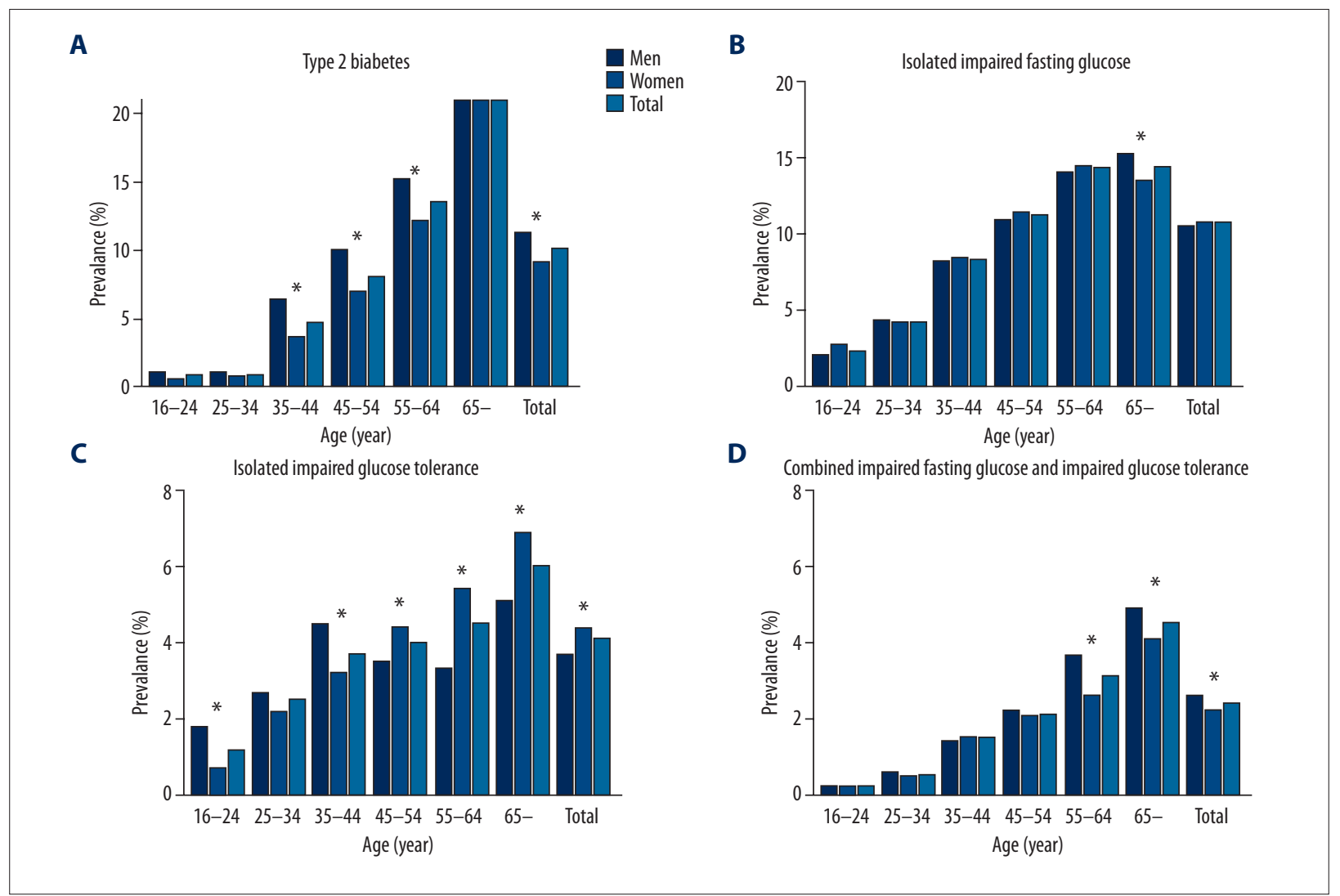

Figure 1. Age-specific prevalence of diabetes mellitus and impaired glucose regulation among men and women. Prevalence of total type 2 diabetes (A), isolated impaired fasting glucose (B), isolated impaired glucose tolerance $(\mathbf{C})$ and combined impaired fasting glucose and impaired glucose tolerance (D) in men and women $\left({ }^{*} P<0.05\right)$.

with missing plasma lipid profile, 14385 (42.8\% men) were included in the final analysis. Of these, 14342 (99.7\%) were ethnic Han Chinese, 5084 subjects were from rural and 9301 were from urban areas, aged $16-88$ years, living in Shanghai.

\section{Prevalence of type 2 diabetes and impaired glucose regulation}

There were 1456 subjects (10.1\%) with type 2 diabetes, including 699 (11.4\%) men and 757 (9.2\%) women (Figure 1). The prevalence of isolated IGF, isolated IGT, and combined IFG and IGT was $10.8 \%, 4.1 \%$ and $2.4 \%$, respectively. After age and sex standardization based on the 2000 China census data, the type 2 diabetes prevalence was $7.0 \%$ (7.6\% for men and $6.7 \%$ for women) (data not shown). Men showed a significantly higher prevalence of diabetes than women $\left(\chi^{2}=18.28, P<0.01\right)$.

Prevalence of type 2 diabetes increased with age, from $0.9 \%$ (15-24 years age group) to $22.5 \%$ (65 years of age and older). The prevalence of type 2 diabetes was $<10 \%$ before the age of 55 years. The prevalence of impaired glucose regulation increased with age.
The prevalence of isolated IFG, type 2 diabetes, and combined IFG and IGT in urban areas was significantly higher than in rural areas $(13.8 \%, 12.8 \%$, and $2.9 \%$, respectively, in urban areas; and $5.2 \%, 5.2 \%$, and $1.3 \%$, respectively, in rural areas; all $P<0.01)$. However, the prevalence of isolated IGT in rural areas was higher than that in urban areas (2.9\% vs. $1.3 \%$, $P<0.01)$ (Table 1).

The prevalence of type 2 diabetes was higher in subjects with hyperlipidemia than that in subjects without hyperlipidemia (16.9\% vs. $7 \%, P<0.01$ ) (Table 2). A similar higher prevalence was observed for isolated IGT $(6.3 \%$ vs. $3.0 \%, P<0.01)$ and combined IFG and IGT ( $3.9 \%$ vs. $1.7 \%, P<0.01)$.

\section{Analysis of risk factors for type 2 diabetes}

The 14385 subjects were divided into diabetes group (1456) and non-diabetes group (12 929). Univariate analysis showed that sex, age, BMI, WHR, waist circumference, FPG, 2-h PG, triglyceride, TC, TC/HDL-C (total cholesterol/HDL cholesterol ratios), and non-HDL-C (non-HDL cholesterol) were associated with diabetes (Table 3). 
Table 1. Comparison of abnormal glucose metabolisms prevalence and serum glucose concentrations between urban and rural residents in Shanghai.

\begin{tabular}{|c|c|c|c|c|c|c|}
\hline \multirow[b]{2}{*}{ Characteristics } & \multicolumn{3}{|c|}{ Rural areas } & \multicolumn{3}{|c|}{ Urban areas } \\
\hline & $\begin{array}{c}\text { Overall } \\
(n=5084)\end{array}$ & $\begin{array}{c}\text { Without } \\
\text { hyperlipidemia } \\
(n=3413)\end{array}$ & $\begin{array}{c}\text { With } \\
\text { hyperlipidemia } \\
(n=1671)\end{array}$ & $\begin{array}{c}\text { Overall } \\
(n=9301)\end{array}$ & $\begin{array}{c}\text { Without } \\
\text { Hyperlipidemia } \\
(n=6389)\end{array}$ & $\begin{array}{c}\text { With } \\
\text { Hyperlipidemia } \\
(n=2912)\end{array}$ \\
\hline $\begin{array}{l}\text { Male, \% } \\
(95 \% \mathrm{Cl})\end{array}$ & $\begin{array}{c}39.5 \\
(38.2,40.8)\end{array}$ & $\begin{array}{c}41.1 \\
(39.4,42.8)\end{array}$ & $\begin{array}{c}36.3 \\
(34.0,38.6)^{\star}\end{array}$ & $\begin{array}{c}44.5 \\
(43.5,45.5)^{\star \star \star}\end{array}$ & $\begin{array}{c}41.5 \\
(40.3,42.7)\end{array}$ & $\begin{array}{c}51.1 \\
(49.3,52.9)^{*}\end{array}$ \\
\hline $\begin{array}{l}\text { Age (years), } \\
\text { mean (SD) }\end{array}$ & $\begin{array}{c}47.0 \\
(14.0)\end{array}$ & $\begin{array}{l}45.0 \\
(14.4)\end{array}$ & $\begin{array}{c}51.7 \\
(11.4)^{*}\end{array}$ & $\begin{array}{c}50.9 \\
(14.7)^{\star \star \star}\end{array}$ & $\begin{array}{c}48.9 \\
(15.3)\end{array}$ & $\begin{array}{c}55.3 \\
(12.3)^{*}\end{array}$ \\
\hline $\begin{array}{l}\text { BMI }\left(\mathrm{kg} / \mathrm{m}^{2}\right) \text {, } \\
\text { mean }(\mathrm{SD})\end{array}$ & $\begin{array}{l}23.6 \\
(3.4)\end{array}$ & $\begin{array}{l}23.0 \\
(3.2)\end{array}$ & $\begin{array}{l}24.9 \\
(3.4)^{*}\end{array}$ & $\begin{array}{c}24.1 \\
(3.5)^{\star \star \star \star}\end{array}$ & $\begin{array}{l}23.6 \\
(3.4)\end{array}$ & $\begin{array}{c}25.4 \\
(3.3)^{*}\end{array}$ \\
\hline $\begin{array}{l}\text { WC }(\mathrm{cm}) \\
\text { mean }(\mathrm{SD})\end{array}$ & $\begin{array}{l}77.8 \\
(9.4)\end{array}$ & $\begin{array}{l}75.6 \\
(8.9)\end{array}$ & $\begin{array}{l}82.3 \\
(9.0)^{*}\end{array}$ & $\begin{array}{c}80.2 \\
(10.2)^{\star * \star}\end{array}$ & $\begin{array}{l}78.2 \\
(9.9)\end{array}$ & $\begin{array}{l}84.6 \\
(9.3)^{\star}\end{array}$ \\
\hline $\begin{array}{l}\text { FPG (mmol/L), } \\
\text { mean (SD) }\end{array}$ & $\begin{array}{c}4.7 \\
(1.4)\end{array}$ & $\begin{array}{c}4.6 \\
(1.1)\end{array}$ & $\begin{array}{c}4.9 \\
(1.8)^{\star}\end{array}$ & $\begin{array}{c}5.5 \\
(1.6)^{\star \star \star}\end{array}$ & $\begin{array}{c}5.3 \\
(1.3)\end{array}$ & $\begin{array}{c}6.0 \\
(2.1)^{*}\end{array}$ \\
\hline $\begin{array}{l}2 \mathrm{~h}-\mathrm{PG}(\mathrm{mmol} / \mathrm{L}) \text {, } \\
\text { mean (SD) }\end{array}$ & $\begin{array}{c}5.7 \\
(2.5)\end{array}$ & $\begin{array}{c}5.3 \\
(2.0)\end{array}$ & $\begin{array}{c}6.5 \\
(2.3)^{*}\end{array}$ & $\begin{array}{c}6.0 \\
(2.9)^{\star \star \star}\end{array}$ & $\begin{array}{c}5.6 \\
(2.4)\end{array}$ & $\begin{array}{c}7.0 \\
(3.7)^{\star}\end{array}$ \\
\hline $\begin{array}{l}\text { IFG, \% } \\
(95 \% \mathrm{CI})\end{array}$ & $\begin{array}{c}5.2 \\
(4.6,5.8)\end{array}$ & $\begin{array}{c}5.4 \\
(4.6,6.2)\end{array}$ & $\begin{array}{c}4.8 \\
(3.8,5.8)\end{array}$ & $\begin{array}{c}13.8 \\
(13.1,14.5)^{\star \star *}\end{array}$ & $\begin{array}{c}13.0 \\
(12.2,13.8)\end{array}$ & $\begin{array}{c}15.4 \\
(14.1,16.7)^{\star \star}\end{array}$ \\
\hline $\begin{array}{l}\text { IGT, \% } \\
(95 \% \mathrm{CI})\end{array}$ & $\begin{array}{c}6.1 \\
(5.4,6.8)\end{array}$ & $\begin{array}{c}4.5 \\
(3.8,5.2)\end{array}$ & $\begin{array}{c}9.6 \\
(8.2,11.0)^{*}\end{array}$ & $\begin{array}{c}3.0 \\
(2.7,3.4)^{\star \star \star}\end{array}$ & $\begin{array}{c}2.3 \\
(1.9,2.7)\end{array}$ & $\begin{array}{c}4.5 \\
(3.7,5.3)^{*}\end{array}$ \\
\hline $\begin{array}{l}\text { IFG+IGT, } \\
\%(95 \% \mathrm{Cl})\end{array}$ & $1.3(1.0,1.6)$ & $1.1(0.8,1.4)$ & $\begin{array}{c}1.8 \\
(1.2,2.4)^{\star *}\end{array}$ & $\begin{array}{c}2.9 \\
(2.6,3.2)^{\star \star \star}\end{array}$ & $\begin{array}{c}2.0 \\
(1.7,2.3)\end{array}$ & $\begin{array}{c}5.1 \\
(4.3,5.9)^{\star}\end{array}$ \\
\hline $\begin{array}{l}\text { Diabetes, } \\
\%(95 \% \mathrm{Cl})\end{array}$ & $\begin{array}{c}5.2 \\
(4.6,5.8)\end{array}$ & $\begin{array}{c}3.5 \\
(2.9,4.1)\end{array}$ & $\begin{array}{c}8.9 \\
(7.5,10.3)^{*}\end{array}$ & $\begin{array}{c}12.8 \\
(12.1,13.5)^{\star \star *}\end{array}$ & $\begin{array}{c}8.8 \\
(8.1,9.5)\end{array}$ & $\begin{array}{c}21.5 \\
(20.0,23.0)^{*}\end{array}$ \\
\hline
\end{tabular}

* P-value $<0.01$ between with and without hyperlipidemia; ${ }^{* *} \mathrm{P}$-value $<0.05$ between with and without hyperlipidemia; ${ }^{* * *} \mathrm{P}$-value $<0.01$ between rural and urban areas. BMI - body mass index; WC - waist circumference; FPG - fasting plasma glucose; $2 \mathrm{~h}-\mathrm{PG}-2-\mathrm{h}$ postprandial plasma glucose; IFG - isolated impaired fasting glucose; IGT - isolated impaired glucose tolerance; IFG+IGT - combined impaired fasting glucose and impaired glucose tolerance.

Multivariable logistic regression revealed that urban residence, age, waist circumference, isolated hypertriglyceridemia, isolated hypercholesterolemia, and mixed hyperlipidemia were independently related to type 2 diabetes. The odds ratio (OR) for type 2 diabetes and the corresponding 95\% confidence intervals $(\mathrm{Cl})$ were calculated using a dichotomous variable logistic regression model (Table 4).

\section{Age- and sex-specific blood glucose categories in hyperlipidemia}

Compared with individuals without hyperlipidemia, levels of FBG and $2 \mathrm{hPG}$ increased in subjects with hyperlipidemia in most of the age groups both in men and women (Figure 2). No differences in average levels of FBG and 2hPG were seen between men and women. The levels of FBG and 2hPG increased with age.
The prevalence of type 2 diabetes increased significantly with increasing BMI ( $P<0.05$ for both hyperlipidemia and without hyperlipidemia) in normal waist circumference subgroups (Figure 3). In each BMI subgroup, the prevalence of type 2 diabetes was higher in central obesity than in normal waist circumference ( $P<0.05$ for both hyperlipidemia and without hyperlipidemia).

\section{Discussion}

The prevalence of type 2 diabetes was reported to be $6.87 \%$ in a sample of 5628 men and women aged 20-94 years, living in a Shanghai urban community in 2001 [5]. This study reports one of the largest population-based type 2 diabetes studies ever conducted in Shanghai. Our investigation demonstrated that the prevalence of type 2 diabetes among Shanghai adults was $10.1 \%$, which is higher than the Chinese national average (9.7\% in 2008) [2]. Furthermore, the prevalence rates of impaired 
Table 2. Comparison of abnormal glucose metabolisms prevalence between with hyperlipidemia and without hyperlipidemia.

Isolated Impaired Fasting Glucose, \% (95\% Cl)

\begin{tabular}{lcc} 
Men & $10.0(9.1,10.9)$ & $11.7(10.3,13.1)^{\star *}$ \\
\hline Women & $10.7(9.9,11.5)$ & $11.4(10.2,12.6)$ \\
\hline Age $\leq 64$ & $9.4(8.8,10.0)$ & $11.2(10.1,12.3)^{*}$ \\
\hline Age $\geq 65$ & $15.7(13.9,17.5)$ & $12.5(10.6,14.4)^{\star *}$ \\
\hline Total & $10.4(9.8,11.0)$ & $11.5(10.6,12.4)^{\star *}$ \\
\hline Isolated impaired glucose tolerance, \% $(95 \% \mathrm{Cl})$ & & $5.2(4.2,6.2)^{\star}$ \\
\hline Men & $2.9(2.4,3.4)$ & $7.4(6.4,8.4)^{\star}$ \\
\hline Women & $3.1(2.7,3.5)$ & $6.2(5.4,7.0)^{\star}$ \\
\hline Age $\leq 64$ & $2.6(2.3,2.9)$ & $6.8(5.3,8.3)$ \\
\hline Age $\geq 65$ & $5.4(4.3,6.5)$ & $6.3(5.6,7.0)^{*}$ \\
\hline Total & $3.0(2.7,3.3)$ &
\end{tabular}

Combined impaired fasting glucose and impaired glucose tolerance, \% $(95 \% \mathrm{Cl})$

\begin{tabular}{lcc}
\hline Men & $1.5(1.1,1.9)$ & $4.7(3.8,5.6)^{\star}$ \\
\hline Women & $1.8(1.5,2.2)$ & $3.2(2.5,3.9)^{\star}$ \\
\hline Age $\leq 64$ & $1.3(1.1,1.5)$ & $3.3(2.7,3.9)^{\star}$ \\
\hline Age $\geq 65$ & $3.6(2.7,4.5)$ & $5.6(4.3,6.9)^{\star *}$ \\
\hline Total & $1.7(1.4,2.0)$ & $3.9(3.3,4.5)^{\star}$ \\
\hline Diabetes, \% $(95 \% \mathrm{Cl})$ & & $17.3(15.7,18.9)^{\star}$ \\
Men & $8.3(7.5,9.1)$ & $16.6(15.1,18.1)^{\star}$ \\
\hline Women & $6.0(5.4,6.6)$ & $13.7(12.6,14.8)^{\star}$ \\
\hline Age $\leq 64$ & $4.6(4.1,5.1)$ & $26.8(24.2,29.4)^{\star}$ \\
Age $\geq 65$ & $19.4(17.4,21.4)$ & $16.9(15.8,18.0)^{\star}$ \\
\hline Total & $7.0(6.5,7.5)$ & \\
\hline
\end{tabular}

* P-value $<0.01$ between with and without hyperlipidemia; ** P-value <0.05 between with and without hyperlipidemia.

fasting glucose and impaired glucose tolerance (17.3\%) found in this study were significantly higher than average Chinese national standards (15.5\%). The prevalence of diabetes was higher among urban than rural residents [2]. Thus, the high incidence of diabetes in Chinese adults prompted screening of the highrisk population for early detection of diabetes.

Patients with hyperlipidemia are at an increased risk of developing type 2 diabetes and abnormal glucose metabolism [4], as well as increased morbidity and mortality from type 2 diabetes and cardiovascular disease $[9,10]$. It is well established that serum lipid profiles are worse in diabetic than in non-diabetic individuals from different ethnic groups $[3,4]$. We found that subjects with hyperlipidemia showed higher FPG and $2 \mathrm{~h}-\mathrm{PG}$ levels than normolipidemic individuals. To the best of our knowledge, the prevalence of type 2 diabetes in the dyslipidemia population has been less studied in China. This might be the first large-scale epidemiologic study on the prevalence of and risk factors for type 2 diabetes in Chinese patients with dyslipidemia in China.

The most common pattern of dyslipidemia in Chinese with and without type 2 diabetes is represented by elevated serum triglyceride levels and hypertriglyceridemia [11]. Hypertriglyceridemia was an independent risk factor for type 2 diabetes in Qingdao, north China [12]. Hypertriglyceridemia (31.8\%) was the most 
Table 3. Anthropometric data and serum glucose and lipid levels in 14,385 participants in Shanghai, China.

\begin{tabular}{|c|c|c|c|c|c|c|c|}
\hline \multirow[b]{2}{*}{ Characteristics } & \multicolumn{5}{|c|}{ With hyperlipidemia ( $n=4583$ ) } & \multirow[b]{2}{*}{$\begin{array}{l}\text { Without } \\
\text { hyperlipidemia } \\
(n=9802)\end{array}$} & \multirow[b]{2}{*}{$P$-value } \\
\hline & $\begin{array}{l}\text { All subjects } \\
(n=14,385)\end{array}$ & $\begin{array}{l}\text { Isolated } \\
\text { hypertrigly- } \\
\text { ceridemia } \\
(\mathbf{n = 3 5 8 2 )}\end{array}$ & $\begin{array}{l}\text { Isolated } \\
\text { hypercholeste- } \\
\text { rolemia } \\
(n=457)\end{array}$ & $\begin{array}{c}\text { Mixed } \\
\text { hyperlipidemia } \\
(n=544)\end{array}$ & Overall & & \\
\hline Male, n (\%) & $\begin{array}{l}6150 \\
(42.8)\end{array}$ & $\begin{array}{l}1736 \\
(48.5)\end{array}$ & $\begin{array}{c}153 \\
(33.5)\end{array}$ & $\begin{array}{c}206 \\
(37.9)\end{array}$ & 2095(34.1) & $\begin{array}{l}4055 \\
(65.9)\end{array}$ & $<0.01$ \\
\hline $\begin{array}{l}\text { Age (years), } \\
\text { mean (SD) }\end{array}$ & $\begin{array}{c}49.5 \\
(14.5)\end{array}$ & $\begin{array}{l}52.7 \\
(12.4)\end{array}$ & $\begin{array}{c}58.5 \\
(10.4)\end{array}$ & $\begin{array}{c}57.8 \\
(10.4)\end{array}$ & $\begin{array}{l}54.0 \\
(12.1)\end{array}$ & $\begin{array}{c}47.6 \\
(15.1)\end{array}$ & $<0.01$ \\
\hline $\begin{array}{l}\text { BMI }\left(\mathrm{kg} / \mathrm{m}^{2}\right) \text {, } \\
\text { mean }(\mathrm{SD})\end{array}$ & $\begin{array}{l}24.0 \\
(3.5)\end{array}$ & $\begin{array}{l}25.3 \\
(3.3)\end{array}$ & $\begin{array}{l}24.1 \\
(3.4)\end{array}$ & $\begin{array}{l}25.8 \\
(3.4)\end{array}$ & $\begin{array}{l}25.2 \\
(3.3)\end{array}$ & $\begin{array}{l}23.4 \\
(3.4)\end{array}$ & $<0.01$ \\
\hline $\begin{array}{l}\text { WC }(\mathrm{cm}) \\
\text { mean }(\mathrm{SD})\end{array}$ & $\begin{array}{c}79.4 \\
(10.0)\end{array}$ & $\begin{array}{l}84.0 \\
(9.1)\end{array}$ & $\begin{array}{l}80.3 \\
(9.9)\end{array}$ & $\begin{array}{l}85.3 \\
(9.2)\end{array}$ & $\begin{array}{l}83.8 \\
(9.2)\end{array}$ & $\begin{array}{l}77.3 \\
(9.7)\end{array}$ & $<0.01$ \\
\hline $\begin{array}{l}\text { WHR, } \\
\text { mean (SD) }\end{array}$ & $\begin{array}{l}0.85 \\
(0.07)\end{array}$ & $\begin{array}{l}0.88 \\
(0.06)\end{array}$ & $\begin{array}{l}0.84 \\
(0.07)\end{array}$ & $\begin{array}{l}0.88 \\
(0.06)\end{array}$ & $\begin{array}{l}0.87 \\
(0.07)\end{array}$ & $\begin{array}{l}0.83 \\
(0.07)\end{array}$ & $<0.01$ \\
\hline $\begin{array}{l}\text { TG (mmol/L), } \\
\text { mean (SD) }\end{array}$ & $\begin{array}{c}1.5 \\
(1.2)\end{array}$ & $\begin{array}{l}2.7 \\
(1.4)\end{array}$ & $\begin{array}{c}1.2 \\
(0.3)\end{array}$ & $\begin{array}{c}3.4 \\
(2.9)\end{array}$ & $\begin{array}{c}2.6 \\
(1.7)\end{array}$ & $\begin{array}{c}1.0 \\
(0.3)\end{array}$ & $<0.01$ \\
\hline $\begin{array}{l}\mathrm{TC}(\mathrm{mmol} / \mathrm{L}) \text {, } \\
\text { mean }(\mathrm{SD})\end{array}$ & $\begin{array}{c}4.7 \\
(1.1)\end{array}$ & $\begin{array}{c}4.8 \\
(0.8)\end{array}$ & $\begin{array}{c}6.8 \\
(1.0)\end{array}$ & $\begin{array}{l}6.9 \\
(0.9)\end{array}$ & $\begin{array}{c}5.2 \\
(1.2)\end{array}$ & $\begin{array}{c}4.4 \\
(0.8)\end{array}$ & $<0.01$ \\
\hline $\begin{array}{l}\text { HDL-C (mmol/L), } \\
\text { mean (SD) }\end{array}$ & $\begin{array}{c}1.4 \\
(0.5)\end{array}$ & $\begin{array}{l}1.3 \\
(0.5)\end{array}$ & $\begin{array}{c}1.8 \\
(0.5)\end{array}$ & $\begin{array}{l}1.5 \\
(0.4)\end{array}$ & $\begin{array}{c}1.3 \\
(0.5)\end{array}$ & $\begin{array}{c}1.4 \\
(0.5)\end{array}$ & $<0.01$ \\
\hline $\begin{array}{l}\mathrm{TC} / \mathrm{HDL} \\
\text { mean }(\mathrm{SD})\end{array}$ & $\begin{array}{c}3.5 \\
(1.2)\end{array}$ & $\begin{array}{c}4.0 \\
(1.2)\end{array}$ & $\begin{array}{c}4.1 \\
(1.3)\end{array}$ & $\begin{array}{c}5.1 \\
(1.7)\end{array}$ & $\begin{array}{c}4.1 \\
(1.3)\end{array}$ & $\begin{array}{c}3.2 \\
(1.0)\end{array}$ & $<0.01$ \\
\hline $\begin{array}{l}\text { non-HDL }(\mathrm{mmol} / \mathrm{L}) \text {, } \\
\text { mean }(\mathrm{SD})\end{array}$ & $\begin{array}{c}3.2 \\
(1.0)\end{array}$ & $\begin{array}{c}3.5 \\
(0.8)\end{array}$ & $\begin{array}{c}5.0 \\
(1.1)\end{array}$ & $\begin{array}{c}5.5 \\
(0.9)\end{array}$ & $\begin{array}{c}3.9 \\
(1.2)\end{array}$ & $\begin{array}{c}2.9 \\
(0.8)\end{array}$ & $<0.01$ \\
\hline $\begin{array}{l}\text { FPG }(\mathrm{mmol} / \mathrm{L}) \text {, } \\
\text { mean }(\mathrm{SD})\end{array}$ & $\begin{array}{c}5.2 \\
(1.6)\end{array}$ & $\begin{array}{c}5.5 \\
(2.0)\end{array}$ & $\begin{array}{c}5.7 \\
(2.0)\end{array}$ & $\begin{array}{c}6.2 \\
(2.5)\end{array}$ & $\begin{array}{c}5.6 \\
(2.1)\end{array}$ & $\begin{array}{c}5.0 \\
(1.3)\end{array}$ & $<0.01$ \\
\hline $\begin{array}{l}2 \mathrm{~h}-\mathrm{PG}(\mathrm{mmol} / \mathrm{L}) \text {, } \\
\text { mean }(\mathrm{SD})\end{array}$ & $\begin{array}{c}5.9 \\
(2.8)\end{array}$ & $\begin{array}{c}6.7 \\
(3.4)\end{array}$ & $\begin{array}{c}6.3 \\
(3.6)\end{array}$ & $\begin{array}{c}7.9 \\
(4.4)\end{array}$ & $\begin{array}{c}6.8 \\
(3.6)\end{array}$ & $\begin{array}{c}5.5 \\
(2.0)\end{array}$ & $<0.01$ \\
\hline $\begin{array}{l}\text { IFG, \% } \\
(95 \% \mathrm{CI})\end{array}$ & $\begin{array}{c}10.8 \\
(10.3,11.3)\end{array}$ & $\begin{array}{c}10.6 \\
(9.6,11.6)\end{array}$ & $\begin{array}{c}14.9 \\
(11.6,18.2)\end{array}$ & $\begin{array}{c}15.3 \\
(12.3,18.3)\end{array}$ & $\begin{array}{c}11.5 \\
(10.6,12.4)\end{array}$ & $\begin{array}{c}10.4 \\
(9.8,11.0)\end{array}$ & 0.037 \\
\hline $\begin{array}{l}\text { IGT, \% } \\
(95 \% \mathrm{CI})\end{array}$ & $\begin{array}{c}4.1 \\
(3.8,4.4)\end{array}$ & $\begin{array}{c}6.9 \\
(6.1,7.7)\end{array}$ & $\begin{array}{c}3.7 \\
(2.0,5.4)\end{array}$ & $\begin{array}{c}4.8 \\
(3.0,6.6)\end{array}$ & $\begin{array}{c}6.3 \\
(5.6,7.0)\end{array}$ & $\begin{array}{c}3.0 \\
(2.7,3.3)\end{array}$ & $<0.01$ \\
\hline $\begin{array}{l}\text { IFG+IGT, } \\
\%(95 \% \mathrm{CI})\end{array}$ & $\begin{array}{c}2.4 \\
(2.1,2.7)\end{array}$ & $\begin{array}{c}3.9 \\
(3.3,4.5)\end{array}$ & $\begin{array}{c}2.2 \\
(0.9,3.5)\end{array}$ & $\begin{array}{c}5.5 \\
(3.6,7.4)\end{array}$ & $\begin{array}{c}3.9 \\
(3.3,4.5)\end{array}$ & $\begin{array}{c}1.7 \\
(1.4,2.0)\end{array}$ & $<0.01$ \\
\hline $\begin{array}{l}\text { Diabetes, \% } \\
(95 \% \mathrm{Cl})\end{array}$ & $\begin{array}{c}10.1 \\
(9.6,10.6)\end{array}$ & $\begin{array}{c}15.3 \\
(14.1,16.5)\end{array}$ & $\begin{array}{c}16.4 \\
(13.0,19.8)\end{array}$ & $\begin{array}{c}27.9 \\
(24.1,31.7)\end{array}$ & $\begin{array}{c}16.9 \\
(15.8,18.0)\end{array}$ & $\begin{array}{c}7.0 \\
(6.5,7.5)\end{array}$ & $<0.01$ \\
\hline
\end{tabular}

a $P$-value for comparison between subjects with normal glucose tolerance or not at $\alpha=0.01$ level. $\mathrm{BMI}-$ body mass index; WC - waist circumference; WHR - waist-to-hip ratio; TC - total cholesterol; TG - triglyceride; HDL-C - high-density lipoprotein cholesterol; TC/HDL - total cholesterol/HDL cholesterol ratios; non-HDL - non-HDL cholesterol (the difference between total cholesterol and high-density lipoprotein cholesterol); FPG - fasting plasma glucose; 2h-PG - 2-h postprandial plasma glucose; IFG - isolated impaired fasting glucose; IGT - isolated impaired glucose tolerance; IFG+IGT - combined impaired fasting glucose and impaired glucose tolerance.

common phenotype of hyperlipidemia in this study, and therefore, a total of 547 diabetics (35.7\%) were diagnosed with hypertriglyceridemia. However, the risk of isolated hypertriglyceridemia and isolated hypercholesterolemia for type 2 diabetes was almost similar (OR 1.7 vs. 1.5) in this study. Our study showed that subjects with mixed hyperlipidemia were more than 3 times higher at risk of developing type 2 diabetes compared with participants with normal lipidemia. Some studies supported the association of serum glucose with TC level, although not as clearly as compared with serum triglyceride level [3]. In addition, isolated low HDL-C showed no effect on the prevalence of type 2 diabetes in this study. 
Table 4. Multivariable-adjusted odds ratios for type 2 diabetes.

\begin{tabular}{|c|c|c|c|c|c|c|}
\hline Variable & Coefficient of regression (b) & Standard error (SE) & Wald $\chi^{2}$ & $\mathbf{P}$ & $\mathbf{O R}^{*}$ & $95 \% \mathrm{Cl}$ \\
\hline Urban residence & 0.721 & 0.074 & 95.199 & 0.000 & 2.056 & $1.779-2.377$ \\
\hline $\begin{array}{l}\text { Age, per } 10-y r \\
\text { increment }\end{array}$ & 0.496 & 0.026 & 356.549 & 0.000 & 1.642 & $1.560-1.729$ \\
\hline Waist circumference & 0.045 & 0.003 & 198.138 & 0.000 & 1.046 & $1.039-1.052$ \\
\hline $\begin{array}{l}\text { Isolated } \\
\text { hypertriglyceridemia** }\end{array}$ & 0.557 & 0.066 & 71.175 & 0.000 & 1.745 & $1.533-1.986$ \\
\hline $\begin{array}{l}\text { Isolated } \\
\text { hypercholesterolemia*** }\end{array}$ & 0.428 & 0.138 & 9.627 & 0.002 & 1.534 & $1.171-2.009$ \\
\hline Mixed hyperlipidemia\# & 1.074 & 0.109 & 97.065 & 0.000 & 2.928 & $2.365-3.626$ \\
\hline
\end{tabular}

* Odds ratios were calculated with the use of dichotomous variable logistic regression model. All covariables listed were included in the model simultaneously. Status with respect to sex and BMI were not significantly associated with the risk of diabetes and were not included in the final model. ** Isolated hypertriglyceridemia was defined as serum triglycerides $\geq 1.7 \mathrm{mmol} / \mathrm{L}$ and total cholesterol $<6.2 \mathrm{mmol} / \mathrm{L}$. *** Isolated hypercholesterolemia was defined as total cholesterol $\geq 6.2 \mathrm{mmol} / \mathrm{l}$ or on medication and triglycerides $<1.7 \mathrm{mmol} / \mathrm{L}$. " Mixed hyperlipidemia was defined as triglycerides $\geq 1.7 \mathrm{mmol} / \mathrm{L}$ and total cholesterol $\geq 6.2 \mathrm{mmol} / \mathrm{L}$.

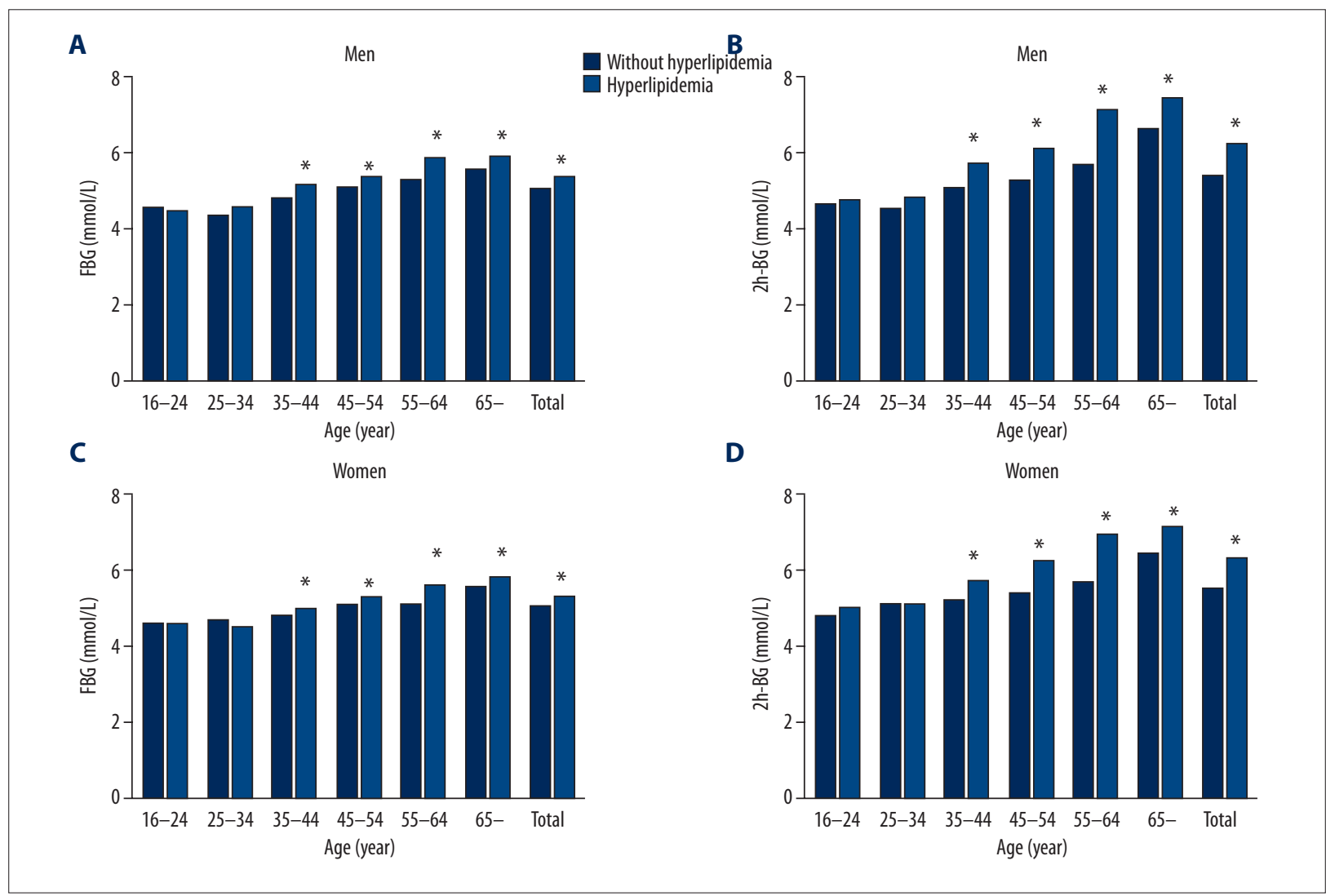

Figure 2. Age- and sex-specific serum glucose levels with hyperlipidemia. Fasting plasma glucose level in men (A), in women (C) and 2-h postprandial plasma glucose in men (B), in women (D) with and without hyperlipidemia $\left({ }^{*} P<0.05\right)$.

Type 2 diabetes and hyperlipidemia are diagnosed within the same individuals, due to common risk factors including age, sex, obesity, physical inactivity, and high fat diet. The prevalence of hyperlipidemia increased with age, which was reported in our previous study [6]. Among patients with hyperlipidemia aged above 35 , prevalence of type 2 diabetes also increased, with a prevalence of $4.7 \%$ in ages 35 to 44 years, $8.1 \%$ in ages 45 to 54 years, $13.5 \%$ in those aged 55 to 64 years, and $22.5 \%$ in 


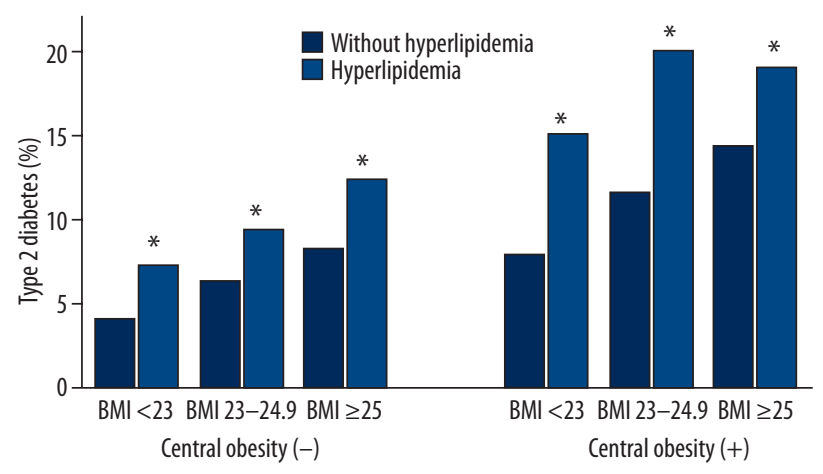

Figure 3. BMI- and waist circumference- specific prevalence of type 2 diabetes with hyperlipidemia. Prevalence of total type 2 diabetes in patients, based on BMI; Central obesity is defined as waist circumference greater than 90 $\mathrm{cm}$ in males and greater than $80 \mathrm{~cm}$ in females. * Statistically different from control group $(P<0.05)$.

those aged 65 years or older. Although the prevalence of hyperlipidemia was higher in men than in women, we observed a similar risk of diabetes between men and women with hyperlipidemia, which was confirmed by multivariate analysis. Although these shared risk factors increased the risk of type 2 diabetes and dyslipidemia, these factors were interrelated and difficult to assess in isolation.

Type 2 diabetes is characterized by both insulin resistance and hyperinsulinemia. In hyperlipidemia patients, the free fatty acids act as precursors to gluconeogenesis in the liver, increase apoptosis in beta cells of the pancreas, and raise insulin resistance in muscles [13], resulting in impaired insulin secretion and persistent hyperglycemia. The biological links between diabetes and dyslipidemia are not clear [14].

Serum lipid values are the predictors of coronary heart disease among East Asians with diabetes. Aggressive interventions in dyslipidemia reduce the risk of cardiovascular event for patients with type 2 diabetes. Recent findings from the Cholesterol Treatment Trialists study using individual patient data in meta-analysis indicate that the benefits of primary prevention with statins include lower ( $<1 \%$ per year) risk of a major cardiovascular event [15]. On the other hand, improved glycemic control is very effective in reducing serum triglyceride levels. Insulin therapy (alone or with insulin sensitizers) may also be particularly effective in lowering serum triglyceride levels in diabetic patients with hyperlipidemia [16]. Endothelial function is associated with complex interaction between dyslipidemia and incident of type 2 diabetes and cardiovascular disease $[17,18]$.

Our study has several limitations. First, previously diagnosed diabetes was defined as self-reported diabetes using a questionnaire, resulting in a potential bias. Second, the time point of diabetes diagnosis was not considered in the study. Finally, we failed to evaluate the role of pharmacological anamnesis and family history of diabetes in development of type 2 diabetes. In China, more than $40 \%$ of patients with type 2 diabetes remain undiagnosed and untreated.

\section{Conclusions}

Our study suggests that type 2 diabetes is very common in hyperlipidemia patients in Shanghai, and both serum levels of triglyceride and TC are associated with FPG and 2h-PG. Therefore, prompt intervention for prevention and treatment of diabetes in patients with hyperlipidemia, especially combined hypertriglyceridemia and hypercholesterolemia, are required. In 2009, the USPSTF concluded that the evidence was insufficient to recommend for or against routinely screening asymptomatic adults for type 2 diabetes or pre-diabetes, but it did recommend screening adults with hypertension or hyperlipidemia $[19,20]$. Screening adults for type 2 diabetes in Chinese patients with hyperlipidemia may be effective, especially in those with mixed hyperlipidemia in China.

\section{Conflicts of interest}

No potential conflicts of interest relevant to this article were reported.

\section{Acknowledgments}

GY.C. wrote the manuscript and researched data. X.X. researched data and contributed to discussion. JG.F. contributed to the discussion and reviewed/edited the manuscript. Many people helped with data collection and entry, including L.L., F.D., YY.L., and XJ.L. 


\section{References:}

1. Ma RC, Lin X, Jia W: Causes of type 2 diabetes in China. Lancet Diabetes Endocrinol, 2014; 2(12): 980-91

2. Yang W, Lu J, Weng J et al: Prevalence of diabetes among men and women in China. N Engl J Med, 2010; 362(12): 1090-101

3. Zhang L, Qiao Q, Tuomilehto J et al: Blood lipid levels in relation to glucose status in seven populations of Asian origin without a prior history of diabetes: the DECODA study. Diabetes Metab Res Rev, 2009; 25(6): 549-57

4. Zhang L, Qiao Q, Tuomilehto J et al: Blood lipid levels in relation to glucose status in European men and women without a prior history of diabetes: the DECODE Study. Diabetes Res Clin Pract, 2008; 82(3): 364-77

5. Jia WP, Pang $C$, Chen $L$ et al: Epidemiological characteristics of diabetes mellitus and impaired glucose regulation in a Chinese adult population: the Shanghai Diabetes Studies, a cross-sectional 3-year follow-up study in Shanghai urban communities. Diabetologia, 2007; 50(2): 286-92

6. Wu JY, Duan XY, Li L et al: Dyslipidemia in Shanghai, China. Prev Med, 2010; 51(5): 412-15

7. American Diabetes Association: Standards of medical care in diabetes 2014. Diabetes Care, 2014; 37(Suppl.1): S14-80

8. Marchesini G, Forlani G, Cerrelli F et al: WHO and ATPIII proposals for the definition of the metabolic syndrome in patients with Type 2 diabetes. Diabet Med, 2004; 21(4): 383-87

9. Cui HB, Wang SH, Wang DQ et al: Modified classic risk factors for coronary artery disease in Chinese Han population. Chin Med Sci J, 2007; 22(4): 216-23

10. Wang SH, Sun ZL, Ruan XZ et al: Dyslipidaemia among diabetic patients with ischemic stroke in a Chinese hospital. Chin Med J, 2009; 122(21): 2567-72

11. Xu H, Song Y, You NC et al: Prevalence and clustering of metabolic risk factors for type 2 diabetes among Chinese adults in Shanghai, China. BMC Public Health, 2010; 10: 683
12. Ning F, Pang ZC, Dong YH et al: Risk factors associated with the dramatic increase in the prevalence of diabetes in the adult Chinese population in Qingdao, China. Diabet Med, 2009; 26(9): 855-63

13. Boden G: Obesity, insulin resistance and free fatty acids. Curr Opin Endocrinol Diabetes Obes, 2011; 18(2): 139-43

14. Chen $\mathrm{H}$, Yu M, Li M et al: Polymorphic variations in manganese superoxide dismutase (MnSOD), glutathione peroxidase-1 (GPX1), and catalase (CAT) contribute to elevated plasma triglyceride levels in Chinese patients with type 2 diabetes or diabetic cardiovascular disease. Mol Cell Biochem, 2012; 363(1-2): 85-91

15. Taylor F, Huffman MD, Macedo AF et al: Statins for the primary prevention of cardiovascular disease. Cochrane Database Syst Rev, 2013; 1: CD004816

16. Aslan I, Kucuksayan E, Aslan M: Effect of insulin analog initiation therapy on LDL/HDL subfraction profile and HDL associated enzymes in type $2 \mathrm{di}-$ abetic patients. Lipids Health Dis, 2013; 12: 54

17. Pannacciulli N, De Pergola G, Ciccone $M$ et al: Effect of family history of type 2 diabetes on the intima-media thickness of the common carotid artery in normal-weight, overweight, and obese glucose-tolerant young adults. Diabetes Care, 2003; 26(4): 1230-34

18. Tavares AC, Bocchi EA, Guimaraes GV: Endothelial function in pre-pubertal children at risk of developing cardiomyopathy: a new frontier. Clinics, 2012; 67(3): 273-78

19. Lin KW, Chang C: Screening for type 2 diabetes mellitus in adults. Am Fam Phys, 2009; 80(10): 1141

20. Norris SL, Kansagara D, Bougatsos C, Fu R, Force USPST: Screening adults for type 2 diabetes: a review of the evidence for the U.S. Preventive Services Task Force. Ann Intern Med, 2008; 148(11): 855-68 